\title{
Beyond one's body parts: Remote object movement with sense of agency involuntarily biases spatial attention
}

\author{
Ryoichi Nakashima ${ }^{1}$
}

Published online: 27 November 2018

(C) Psychonomic Society, Inc. 2018

\begin{abstract}
Humans' own body postures and/or actions bias visual attention. This study examined whether an object controlled remotely with a sense of agency (SoA) can bias attentional allocation, even if the mappings of body movement and object movement do not correspond (i.e., with a less extended body representation). In the experiments, participants shifted a circle toward an instructed direction via keypress. On some trials, the task changed to identification of visual characters instead of the circle shift. Strength of SoA was manipulated based on the probability that the circle actually moved to the participant's intended location, with high probability producing a strong SoA. Results showed that visual identification performance became higher for appearance of the target at the intended location than at the unintended location, but only when observers felt a strong SoA. This suggests that the object's movement attracts attention when the observer feels a strong SoA over its control.
\end{abstract}

Keywords Sense of agency $\cdot$ Remote-controlled object $\cdot$ Visual perception $\cdot$ Spatial attention

The position of body parts (the own body representation) modulates human visual perception, biasing visual attention, even if their position is task irrelevant (e.g., Garza, Strom, Wright, Roberts, \& Reed, 2013; Nakashima \& Kumada, 2017). Further, visual perception is influenced by the position of a hand tool that one holds (e.g., Reed, Betz, Garza, \& Roberts, 2010). Recent studies reported that visual perception on the location of a remote-controlled object other than one's body could be facilitated, such as a live video image of one's hands on a screen (Whiteley, Kennett, Taylor-Clarke, \& Haggard, 2004), virtual hands in a virtual reality space (Short \& Ward, 2009), and a mouse cursor on a display (Gozli \& Brown, 2011). These studies indicated that the spatial correspondence between the controlled object and the manipulator's body part movements (e.g., a cursor moves toward the left when the manipulator shifts the mouse toward the left)

Electronic supplementary material The online version of this article (https://doi.org/10.3758/s13423-018-1552-4) contains supplementary material, which is available to authorized users.

Ryoichi Nakashima rnaka@L.u-tokyo.ac.jp

1 Department of Psychology, Graduate School of Humanities and Society, The University of Tokyo, 7-3-1 Hongo, Bunkyo-Ku, Tokyo 113-0033, Japan is important for facilitating perception via the extended body representation.

Visual attention is usually directed to the action goal when body action (movement) is performed (Deubel, Schneider, \& Paprotta, 1998; see also Baldauf, Wolf, \& Deubel, 2006). Controlling a nonbody object reveals similar effects. For example, when steering a car, drivers orient their gaze toward the desired future path - the intended direction of motion (Mars \& Navarro, 2012). Thus, during action, the location of directed attention can be determined by the action intention. These studies usually postulate that the actual body or object movement usually corresponds to the action intention.

It is well established that the controlled object position and movement influence visual perception via the extended body representation when the spatial mappings correspond between the object and body movements. However, the object movement often differs from the manipulator's movement. For example, while playing videogames, one pushes a button and the character jumps. This type of noncorrespondence sometimes occurs in remote-control tool use (e.g., operating a robot, playing a claw machine game). When spatial mappings do not correspond, it is necessary to create a new corresponding mapping through a lot of practice. Thus, the extended body representation cannot be used immediately, at least not in the same way as the own body representation. In this situation, 
how is visual perception, especially visual attention, modulated based on the movement of remote-controlled objects?

A person using a tool usually feels that she or he is the agent controlling the tool. This feeling is referred to as "sense of agency" (SoA; e.g., Haggard, 2017). In this study, SoA is defined as the feeling that one controls an object as expected rather than the knowledge that one is actually causing the object's movement. The comparator model, which explains why one feels an SoA (e.g., Blakemore, Frith, \& Wolpert, 1999), indicates that when a person intends to act, an outcome is predicted based on her or his motor command and compared with the actual outcome; a match implies the experience of SoA. Thus, when one intends to move an object and the predictability of object movement is high (low), SoA becomes strong (weak).

This study examined the effect of SoA in controlling a remote object on attentional bias when the mappings of body and object movements do not correspond. In this situation, the extended body representation, supported by an SoA, cannot be used perfectly like the own body representation. Thus, rather than SoA itself, either intention alone without the actual outcome, or the predictability of the outcome regardless of the intention may be important for attentional bias. I examined whether intention to control and/or predictability of outcome are important for attracting attention. To accomplish this, an experimental task was developed in which object-control trials and visual-perception trials were mixed within a single block.

\section{Experiment 1}

Experiment 1 examined whether attention is biased toward the direction of object movement when observers control it, even if the mappings of body movement and object movement do not correspond. Particularly, I examined the attentional bias when the object movement provides no information about the visual perception task (i.e., the location of a target to be identified). I manipulated the probability that the object would move to the location where observers intended to move it, assuming that observers would feel a strong SoA when the probability that the object moves as intended was high.

To distinguish between the effect of control intention and outcome predictability, I set three blocked conditions. First, in the high-SoA (hSoA) condition, participants moved an object by themselves, and it moved as they expected (intention with high predictability). Second, in the low-SoA (ISoA) condition, they moved an object by themselves, but it moved randomly (intention with low predictability). Third, in the no-control (NC) condition, they took no action, but the object moved as they expected (no intention with high predictability). This condition is somewhat similar to the endogenous cueing task (e.g., Posner, 1980), where a circle moving in a constant direction can function as a cue pointing to a given location. If the intention to move the object is important for attentional bias, attention would be biased toward the goal of the object movement in the hSoA and ISoA conditions. If the outcome that the object moves toward a given location is important regardless of intention, attention would be biased to where the object frequently moves in the hSoA and $\mathrm{NC}$ conditions. Otherwise, if participants' SoA over controlling the object as expected is important, attention would be biased toward the object movement goal in only the hSoA condition.

\section{Method}

Participants There were 24 participants (20-32 years old), all with normal or corrected-to-normal vision. This study was approved by the Institutional Review Board of the University of Tokyo, and written informed consent was obtained from all participants.

Apparatus Stimulus presentation and data collection were performed by a computer using MATLAB with Psychtoolbox (Brainard, 1997; Pelli, 1997). Stimuli were displayed on a 27-inch liquid crystal display $(2560 \times 1440$ pixels, $60 \mathrm{~Hz})$. Participants responded using a standard keyboard and a standard 10-key pad.

Stimuli and procedure Participants were tested individually. The viewing distance $(57 \mathrm{~cm})$ was fixed using a chin rest. Upon entering the experimental room, participants' keyboard displayed an arrow pointing either left or right on the space bar. The direction of this arrow was counterbalanced between participants. Participants were instructed to press the space bar to move the circle from the center of the display toward the direction indicated by the arrow. This task was repeated throughout the experiment (i.e., an object-control trial). At the end of each experimental block, participants were asked to orally rate the SoA for controlling the circle during that block. They were also told that, in some trials, a keypress would be followed by presentation of two characters (rotated $T$ and $L$ ), instead of the circle, in the display's left and right areas. In such trials, they were asked to identify the orientation of the rotated $T$ (i.e., a visual-perception trial).

Regardless of trial type, each trial began with a white circle $\left(1.5^{\circ} \times 1.5^{\circ}\right)$ located at the center of the uniform gray display (see Fig. 1), and participants were told to gaze at the center of the display during each trial. In an object-control trial, $400 \mathrm{~ms}$ after their keypress (i.e., pressing the space bar), the circle vanished and reappeared $10^{\circ}$ to the left or right of the center. The circle remained visible for $500 \mathrm{~ms}$. Next, after $500 \mathrm{~ms}$ of blank-display presentation, a circle appeared in the center. Participants repeated this sequence.

During visual-perception trials, the circle vanished $400 \mathrm{~ms}$ after a keypress, and $T\left(90^{\circ}\right.$ to the left or right of vertical 
(a) Object-control trial

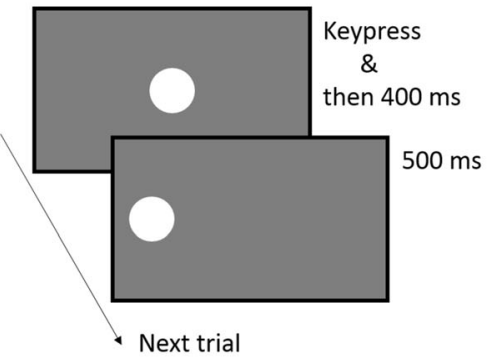

Fig. 1 Examples of sequence of trial in Experiment 1: An object-control trial where the circle moved toward the left (a), and a visual-perception trial where the target stimulus appeared at the left of the display (target orientation: left) (b). Displays were identical at the onset of these trial

center, $0.8^{\circ} \times 0.8^{\circ}$ ) and $L$ (rotated $0^{\circ}, 90^{\circ}, 180^{\circ}$, or $270^{\circ}, 0.8^{\circ}$ $\times 0.8^{\circ}$ ) appeared $10^{\circ}$ to the left and right of the center for 50 $\mathrm{ms}$, before mask stimuli appeared for $500 \mathrm{~ms}$. In these trials, the response display was presented, and participants identified whether the bottom of the $T$ was pointed to the right or left as accurately as possible by keypress on the 10-key pad. To confirm that their gaze was directed to the center of the display, some catch trials were inserted where the $T$ to be identified and then the mask stimulus appeared at the center of the display.

In each experimental block, participants completed a sequence of 110 object-control trials and 50 visual-perception trials. This proportion of trial types was chosen to ensure that participants felt that they were primarily conducting the object-control task. The first 10 trials of each block were object-control trials, and the two trial types were randomly interspersed thereafter. In the 50 visual-perception trials, $T$ and $L$ appeared at the right and left during 40 trials ( $2 T$ orientations $\times 2 T$ locations $\times 10$ repeats), and $T$ appeared in the center of the display during 10 trials ( $T$-orientation was randomly determined). Thus, the direction of circle movement did not predict the target location. After an experimental block, participants provided an oral rating of how strongly they felt that they had controlled the circle as they expected during that block (from $0=$ not at all to $10=$ completely).

The probability that the circle moved to the location where participants intended to move it was manipulated among blocks. In the hSoA (1SoA) block, the circle moved to the intended location during $90 \%$ (50\%) of object-control trials. In the $\mathrm{NC}$ block (160 trials), participants merely viewed the moving circle, which moved to the instructed location in $90 \%$ of trials, without the keypress, and they identified the target in some trials. In this block, they were aware that the keyboard was unplugged from the computer. The visual perception (VP) block (50 trials) included only visual-perception trials (the (b) Visual-perception trial

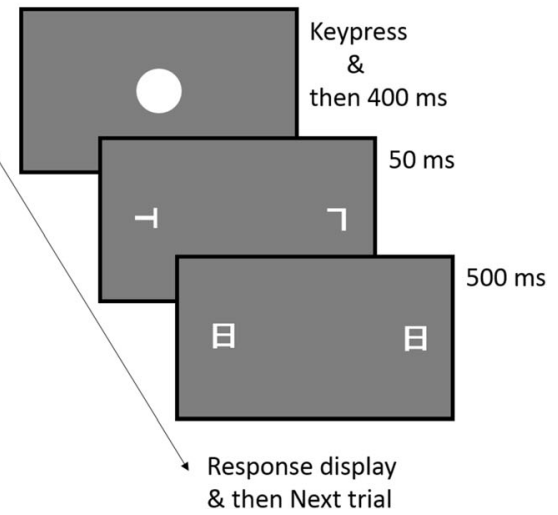

types. These trials were interspersed within one block, and the number of object-control trials was approximately twice that of visual-perception trials. In Experiment 2, a red or green circle was presented on the display instead of a white circle

baseline condition). Ratings were not obtained following NC and VP blocks, because participants did not have any control over the circle's movement. Block order was randomized across participants.

\section{Results}

Subjective ratings of control over the circle were $7.17 \pm 0.34$ (mean $\pm S E$ ) and $3.88 \pm 0.43$ in the hSoA and the 1 SoA blocks respectively, $t(23)=8.20, p<.001$. Thus, manipulation of SoA was successful. Visual-perception trials with a longer reaction time than 4 seconds were excluded from the analysis as outliers (1.6\% of all trials). When the target appeared at the center, target identification performances were above $93 \%$ in all blocks, $F(3,69)=1.04, p=.381$. Thus, it is assumed that participants gazed at the center of the display during a trial, or at least did not gaze at the left or right area beforehand.

Figure 2a summarizes performances in Experiment 1. An analysis of variance (ANOVA) was applied to performance with two factors of block type (hSoA, 1SoA, NC, and VP) and target location (intended and unintended locations). The intended location indicates the location where participants intended to, based on the instruction, move the circle via keypresses, and the unintended location indicates the opposite location. The interaction was significant, $F(3,69)=5.52, p=$ $.002, \eta_{\mathrm{p}}{ }^{2}=.194$. Performance was better at the intended location than at the unintended location during the hSoA block, $p$ $<.001$, whereas no differences during other blocks, $p \mathrm{~s}>.18$. This difference produced the significant main effect of target location, $F(1,23)=5.55, p=.027, \eta_{\mathrm{p}}{ }^{2}=.195$, but nonsignificant effect of block type, $F<1$.

To examine the effect of object control on visual identification, I calculated the difference in performance at each location between each experimental block and the VP block, named the "bias index" (see Fig. 2b). In the hSoA block, the 
(a)

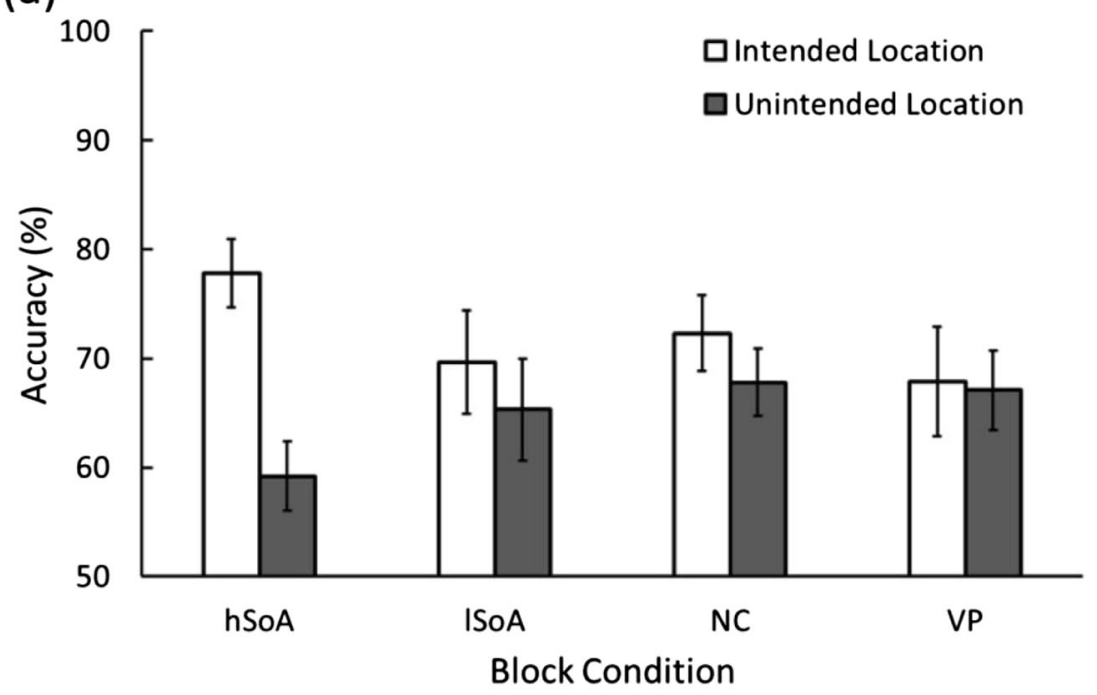

(b)

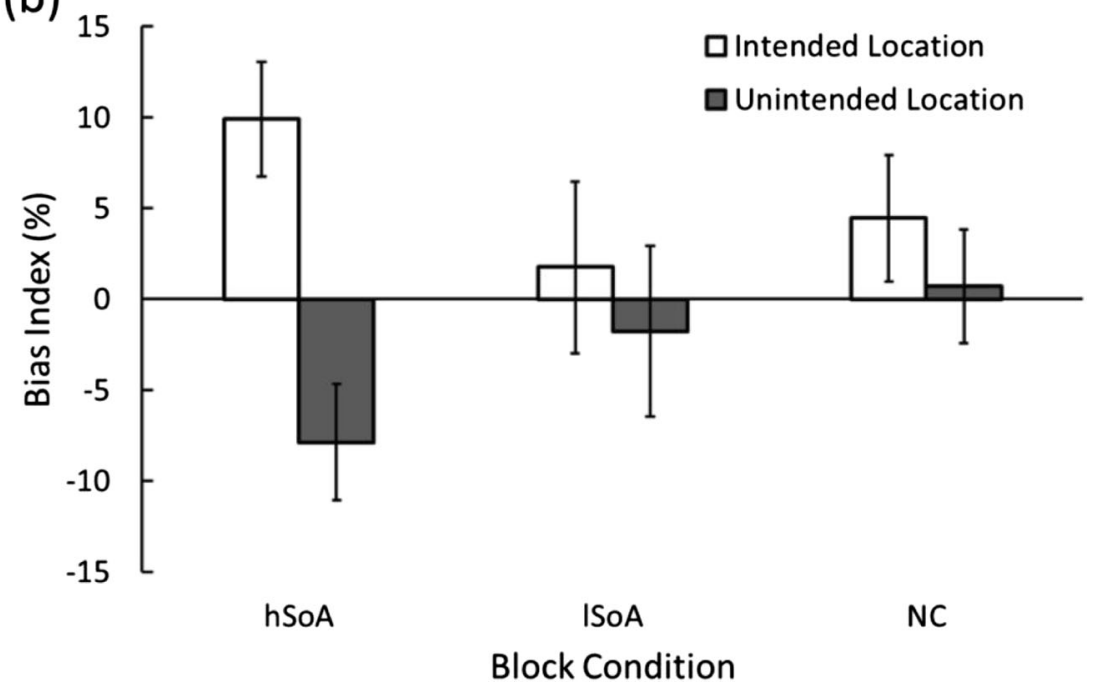

Fig. 2 Summary of the results of Experiment 1: Accuracy (a) and bias index (difference in accuracy of each block relative to that of the VP block) (b) as a function of block and target location. The intended and unintended locations indicate the location where participants intended to

bias index values (the positive values indicate the facilitation of identification) significantly differed from zero, intended location: $t(23)=2.57, p=.017$; unintended location: $t(23)=$ $2.63, p=.015$, whereas this was not the case in the other blocks, $t \mathrm{~s}<1.11$.

\section{Discussion}

In the VP block, performances were not different between the target locations, indicating no bias under typical conditions. Such a conclusion seems reasonable because the target appeared equally at each location.

When the observers pressed a key, a higher probability that a circle moves to their intended location engenders a higher move the circle, and the opposite location, respectively. A positive value for bias index indicates facilitation of visual perception at the location. Error bars indicate $95 \%$ confidence intervals

SoA for controlling the circle. Visual identification performance increased with appearance of the target at the intended location, but only when the observers felt a strong SoA. Further, compared with the general case, when observers feel a strong SoA, visual perception is facilitated in the intended location but inhibited in the unintended location. This is a typical result of attentional processing, since facilitation and inhibition occur simultaneously (e.g., Montagna, Pestilli, \& Carrasco, 2009; Posner, 1980). Thus, a moving object with agency can spatially bias visual attention.

Additionally, performances did not differ between the two locations when observers merely viewed the circle moving without controlling it. These data contradict any assertion that attention is spatially biased based on the fact that the visual 
stimulus appears frequently at a given location (i.e., high predictability). This is consistent with the suggestion that although the endogenous cue could indeed shift attention (Hommel, Pratte, Colzato, \& Godijn, 2001), the effect is relatively small (Ristic \& Kingstone, 2006), especially with short cue-target delay. Thus, direct involvement in controlling the object is important for attentional bias.

The attentional bias induced by object control requires both of the following factors: the observer's intention and actual action of controlling the object, and the outcome of the object moving as intended (i.e., high predictability). Either one alone is not sufficient to create this attentional bias. The binding of the intended action and its outcome is the basis of the SoA. This binding makes observers feel an SoA and biases attention toward the location where they intend to move it.

\section{Experiment 2}

Because the strength of the SoA was manipulated between blocks in Experiment 1, it is possible that global contextual information of the object moving as intended throughout the block produced the attentional bias, rather than the observer's feeling that the object itself moves as intended (i.e., the SoA for controlling the object itself). To examine this possibility, Experiment 2 manipulated the SoA within a block. One of two discriminable objects (i.e., hSoA and 1 SoA objects) appeared in each trial, and participants controlled the object in the trial. Overall, the objects moved toward the intended location with relatively high probability in one block. If global context is critical in inducing attentional bias, attentional bias would be obtained regardless of object type. However, if object control with a strong SoA is critical, attentional bias would be obtained only when the hSoA object appeared.

\section{Method}

There were new 24 participants (19-25 years old). The experimental procedure was the same as that in Experiment 1, with three notable differences. First, there were two experimental blocks: the SoA block and the VP block. Second, in the SoA block (320 trials), a red or green circle, instead of a white circle, appeared at the center in each trial, and trial order was randomized. The first 20 trials of the block were objectcontrol trials (each color circle appeared in 10 trials). One color represented the $\mathrm{hSoA}$ object, moving to the intended location $90 \%$ of the time, while the other circle color represented the 1SoA object (50\%). Overall, the circles moved toward the intended location in $70 \%$ of the object-control trials. The assignment of a color to the SoA object was counterbalanced across participants. Along with this manipulation, in the VP block (100 trials), a red or green circle was presented as a fixation object. Third, participants reported subjective ratings for controlling red and green circles separately after the SoA block.

\section{Results and discussion}

Participants' subjective ratings for controlling the hSoA and the 1 SoA objects were $7.46 \pm 0.24$ and $4.38 \pm 0.25$, respectively, $t(23)=10.06, p<.001$. I omitted $0.8 \%$ of the trials from the analysis. The central target identification performances were above $93 \%, F \mathrm{~s}<1.36, p \mathrm{~s}>.254$.

Figure 3 summarizes the results of Experiment 2. An ANOVA was performed on performance with three factors: block (SoA and VP), object type (hSoA and 1SoA), and target location. The three-way interaction was significant, $F(1,23)=$ 10.91, $p=.003, \eta_{\mathrm{p}}{ }^{2}=.322$. In the SoA block, performance was higher at the intended location than at the unintended location when the hSoA object appeared, $p<.001$, whereas there was no difference when the 1SoA object appeared, $p=$ .596. In the VP block, there was no difference between conditions, $p s>.317$. For the hSoA object, the bias index values were significantly different from zero, intended location: $t(23)$ $=2.10, p=.047$; unintended location: $t(23)=2.82, p=.010$. Meanwhile, the index values for the 1 SoA object were not different from zero, $t \mathrm{~s}<1.02$.

Despite within-block manipulation of the probability that keypresses influenced circle movement, participants felt a strong SoA only for the circle that moved as they intended with high probability. Importantly, attention was biased toward the object movement location when the hSoA object appeared and they intended to control it. This indicates that the observer's recognition of the object type contributes to the attentional bias by control of the object. An additional interpretation is that the effect of the object with agency on attention does not transfer to the other object existing in the same context. Thus, this effect can arise only while feeling agency for control of the object.

\section{General discussion}

This study examined whether visual attentional processing is spatially modulated when observers feel that they control the object spatially as intended (i.e., feeling an SoA). According to the experimental results, visual attention was spatially biased toward the location where the observers intended to move the object only when they felt a strong SoA. This attentional bias requires both the intended action of controlling the object and high predictability of the object actually moving as intended. This study suggests that the SoA for the object controlled remotely influences visual attention. Importantly, this bias occurs when the spatial mappings of body action and object movement do not correspond (e.g., pressing a key and the object moving to the left), in addition to when a body 


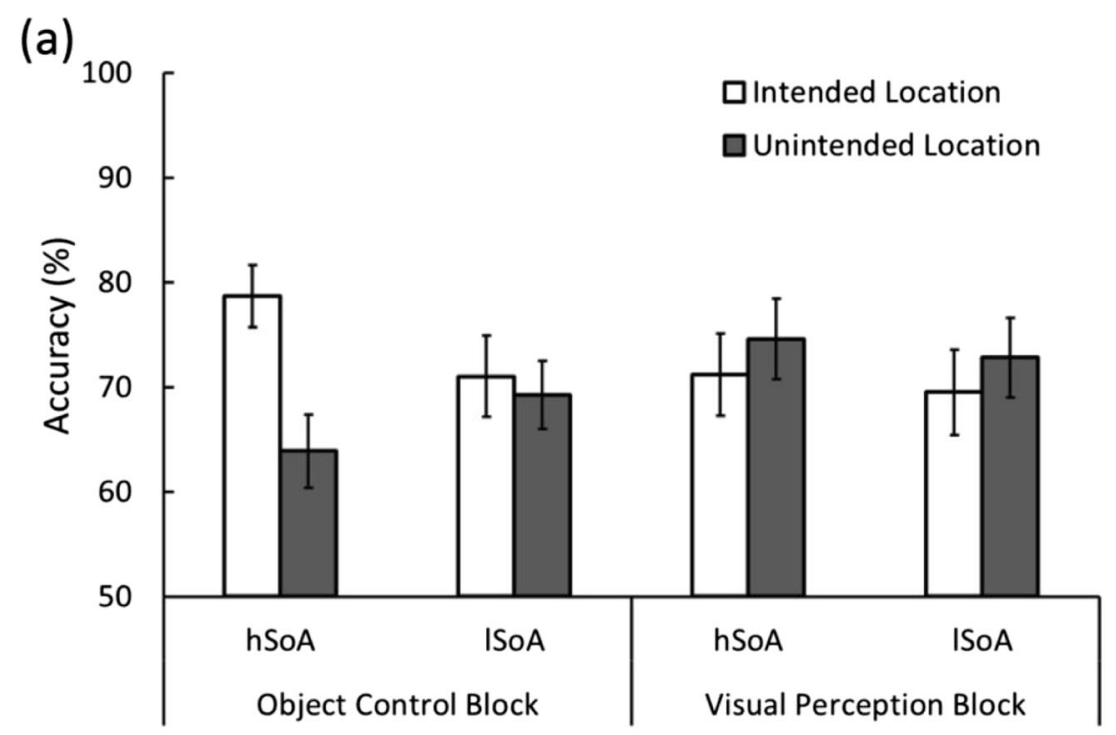

(b)

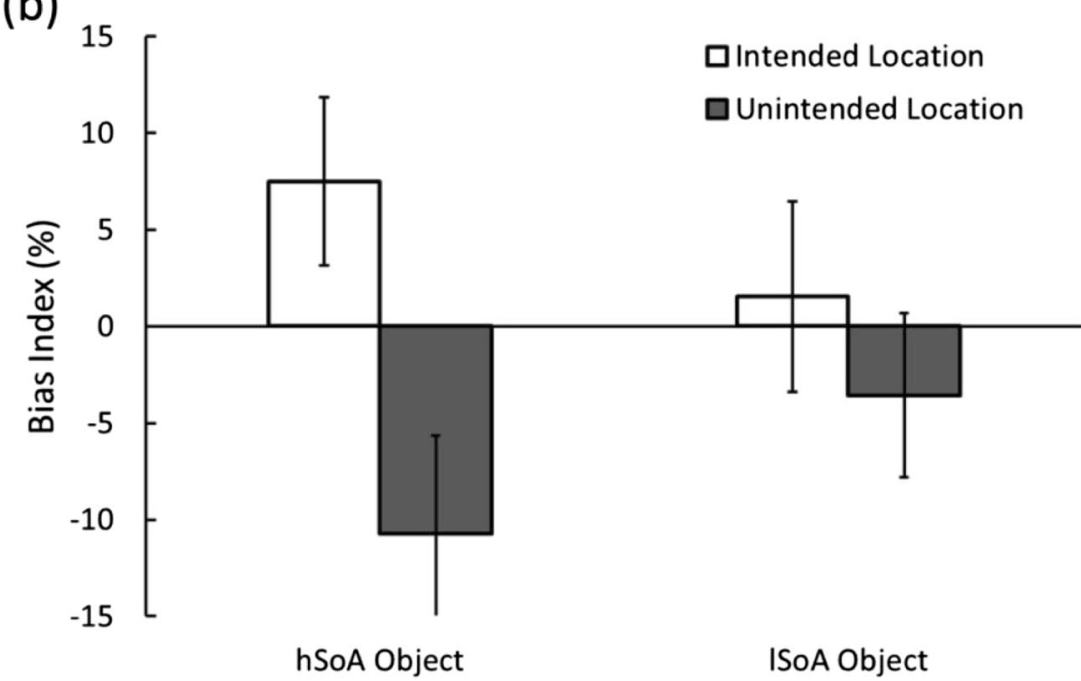

Fig. 3 Summary of the results of Experiment 2: Accuracy (a) and bias index (b) as a function of object type and target location. Error bars indicate 95\% confidence intervals

representation or extended body representation can be used (i.e., when the mappings correspond; Deubel et al., 1998; Short \& Ward, 2009).

Here, I discuss a possible mechanism underlying the spatial attentional bias occurring with the SoA. Combined the comparator model (Blakemore et al., 1999) with the experimental arrangement used in this study, when observers pressed a key to move a circle, they predicted the circle movement outcome based on the intention, and this prediction may shift attention. When the circle moves to the intended location with a high probability, because of the high validity of directing attention to the predicted location, visual perception should be facilitated at the location. That is, the experience of a match between prediction and actual outcome produces the attribution of the object to herself or himself (i.e., an SoA), and simultaneously biases attention toward the location of the outcome.
This study demonstrated that the observer's sustained feeling of agency - rather than brief feelings of agencyinfluences visual attentional processing. In daily situations involving remote tool using, such as operating a robot or playing a video game, a manipulator exerts continued control over the object. The present results are therefore important for understanding visual perception in daily tool-controlling situations. This can be further applied to visual perception during human-computer interactions (e.g., Limerick, Coyle, \& Moore, 2014).

In both experiments, the timing of stimulus appearance in visual-perception trials and circle appearance in object-control trials were always the same. Due to this experimental manipulation, this study can only suggest that attention is directed toward the intended location at the moment when the observer assumes that the object appeared at the location. To examine 
the relationship between SoA and visual attention in detail, it is important to examine the dynamics of attentional shift (i.e., temporal changes in attention; cf. Müller \& Rabbitt, 1989) during feeling an agency.

The near-body advantage for visual perception is not only in biasing attention spatially. The temporal resolution of visual processing increases near the hand via increased activity of the magnocellular pathway (Goodhew, Gozli, Ferber, \& Pratt, 2013). Additionally, learning of action-outcome association influences attentional processing in a relatively complex manner; when the association defines the target, it facilitates target selection, whereas when the association defines the distractor, it facilitates ignoring the distracting stimulus (Gozli, Aslam, \& Pratt, 2016). In light of these recent findings, it is necessary to clarify the effect of an SoA for controlling a remote object (and/or action-outcome association) on visual processing, and to precisely examine the similarity and difference of the effect between full and less extended body representation.

In summary, this study suggests that object movement attracts spatial attention when observers feel a strong SoA for controlling the object in space. Importantly, even when the mappings of body and object movements do not correspond, such as with the less extended body representation, attention is biased toward the location where the observer intends to move it. This contributes to the understanding of visual perception during a variety of remote-object controlling situations. This study therefore makes important suggestions, both theoretically and practically.

Acknowledgements This study was supported by JSPS KAKENHI Grant Number JP16K17368.

Publisher's Note Springer Nature remains neutral with regard to jurisdictional claims in published maps and institutional affiliations.

\section{References}

Baldauf, D., Wolf, M., \& Deubel, H. (2006). Deployment of visual attention before sequences of goal-directed hand movements. Vision Research, 46, 4355-4374.

Blakemore, S.-J., Frith, C. D., \& Wolpert, D. M. (1999). Spatio-temporal prediction modulates the perception of self-produced stimuli. Journal of Cognitive Neuroscience, 11, 551-559.

Brainard, D. H. (1997). The Psychophysics Toolbox. Spatial Vision, 10, 443-446.
Deubel, H., Schneider, W. X., \& Paprotta, I. (1998). Selective dorsal and ventral processing: Evidence for a common attentional mechanism in reaching and perception. Visual Cognition, 5, 81-107.

Garza, J. P., Strom, M. J., Wright, C. E., Roberts, R. J., Jr., \& Reed, C. L. (2013). Top-down influences mediate hand bias in spatial attention. Attention, Perception, \& Psychophysics, 75, 819-823.

Goodhew, S. C., Gozli, D. G., Ferber, S., \& Pratt, J. (2013). Reduced temporal fusion in the near-hand space. Psychological Science, 24, 891-900.

Gozli, D. G., Aslam, H., \& Pratt, J. (2016). Visuospatial cueing by selfcaused features: Orienting of attention and action-outcome associative learning. Psychonomic Bulletin \& Review, 23, 459-467.

Gozli, D. G., \& Brown, L. E. (2011). Agency and control for the integration of a virtual tool into the peripersonal space. Perception, 40, $1309-1319$.

Haggard, P. (2017). Sense of agency in the human brain. Nature Reviews Neuroscience, 18, 197-208.

Hommel, B., Pratt, J., Colzato, L., \& Godijn, R. (2001). Symbolic control of visual attention. Psychological Science, 12, 360-365.

Limerick, H., Coyle, D., \& Moore, J.W. (2014). The experience of agency in human-computer interactions: A review. Frontiers in Human Neuroscience, 8(643). doi:https://doi.org/10.3389/fnhum.2014. 00643.

Mars, F., \& Navarro, J. (2012). Where we look when we drive with or without active steering wheel control. PLOS ONE, 7(8), e43858. doi:https://doi.org/10.1371/journal.pone.0043858

Montagna, B., Pestilli, F., \& Carrasco, M. (2009). Attention trades of spatial acuity. Vision Research, 49, 735-745.

Müller, H. J., \& Rabbitt, P. M. (1989). Reflexive and voluntary orienting of visual attention: Time course of activation and resistance to interruption. Journal of Experimental Psychology: Human Perception and Performance, 15, 315-330.

Nakashima, R., \& Kumada, T. (2017). The whereabouts of visual attention: Involuntary attentional bias toward the default gaze direction. Attention, Perception, \& Psychophysics, 79, 1666-1673.

Pelli, D. G. (1997). The VideoToolbox software for visual psychophysics: Transforming numbers into movies. Spatial Vision, 10, 437-442.

Posner, M. I. (1980). Orienting of attention. The Quarterly Journal of Experimental Psychology, 32, 3-25.

Reed, C. L., Betz, R., Garza, J. P., \& Roberts, R., Jr. (2010). Grab it! Biased attention for functional hand and tool space. Attention, Perception, \& Psychophysics, 72, 236-245.

Ristic, J., \& Kingstone, A. (2006). Attention to arrows: Pointing to a new direction. The Quarterly Journal of Experimental Psychology, 59, 1921-1930.

Short, F., \& Ward, R. (2009). Visual limbs and body space: Critical features for the distinction between the body space and near-body space. Journal of Experimental Psychology: Human Perception and Performance, 35, 1092-1103.

Whiteley, L., Kennett, S., Taylor-Clarke, M., \& Haggard, P. (2004). Facilitated processing of visual stimuli associated with the body. Perception, 33, 307-314. 